\title{
Lens surgery in infancy and childhood
}

\author{
Stephen Hing, Lynne Speedwell, David Taylor
}

\begin{abstract}
The medical records of 224 children who had lens surgery performed at the Hospital for Sick Children during the 10-year period 1978-88 were reviewed. There were 12 children with ectopia lentis; these patients achieved a mean postoperative acuity of $0.68(6 / 8.8)$, and no operative or postoperative complications were noted. Two hundred and twelve children had lens surgery for cataracts or for retrolental abnormalities with a clear lens. Children with bilateral congenital cataracts achieved a mean acuity in the better eye of $0.32(6 / 18)$. In a series of carefully controlled unilateral congenital cataracts the visual results were poor. The postoperative complication rate for cataract and clear lens surgery was $4 \cdot 6 \%$. Because visual results are probably better with early surgery we emphasise that every newborn baby should be tested for media opacities.
\end{abstract}

The commonest reason for operating on the lens in infancy and childhood is to remove a cataract, but sometimes a clear lens is removed if the anterior chamber is flat or shallow and glaucoma is present or threatening - for example, in some cases of persistent hyperplastic primary vitreous (PHPV) and retrolental fibroplasia (RLF). The

TABLE I Type of surgery in 212 patients

\begin{tabular}{lc}
\hline Lensectomy & \\
Bilateral & 80 patients \\
Unilateral & 52 patients \\
Lensectomy in one eye aspiration in the other & 25 patients \\
Total & 237 procedures \\
Aspiration & 29 patients \\
Bilateral & 26 patients \\
Unilateral & 25 patients \\
Aspiration in one eye lensectomy in the other & 109 procedures \\
Total & 346 \\
Total number of primary procedures & \\
\hline
\end{tabular}

TABLE II Mean age at presentation in congenital cataract

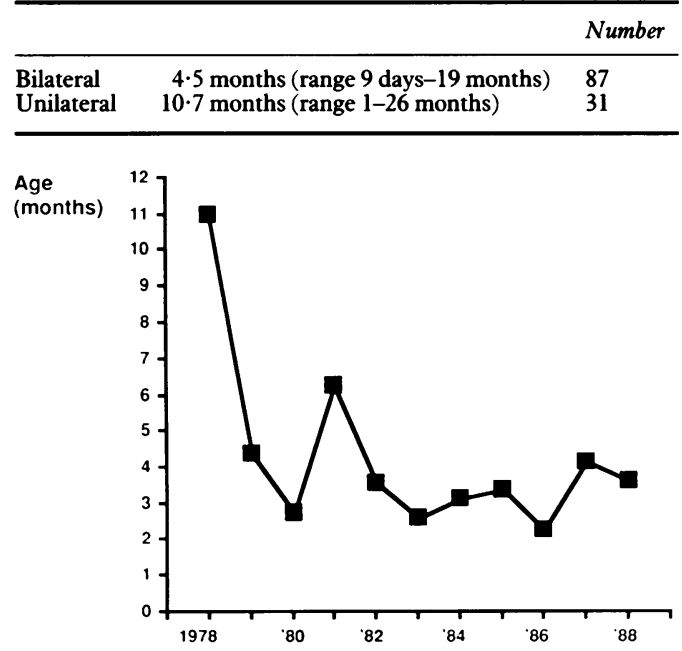

Figure 1: Mean age at presentation to the clinic of infants with bilateral congenital cataract between 1978 and 1988. other reason for operating on the lens in childhood is dislocation of the lens.

In this paper we report the results of a retrospective study of lens surgery at the Hospital for Sick Children and discuss the management.

\section{Material and methods}

The medical records of children who underwent lens surgery at our institution between July 1978 and June 1988 were examined. Most cases were under regular review by us, others were reviewed specially, and information was requested from the patient's current ophthalmologist where one could be identified.

The visual acuities quoted are Snellen line acuities; as they were tested at various distances, they have been converted to decimal fractions and averaged. Children who were unable to do the Snellen vision test reliably were not included in the averaged visual results.

\section{Results}

The records of 224 children were available. Of these, 12 had surgery for ectopia lentis and the rest had either cataracts or clear lenses associated with retrolental abnormalities.

\section{ECTOPIA LENTIS}

Of the 12 children six were cases of simple ectopia lentis, four had Marfan's syndrome (three with a positive family history), and two children had homocystinuria (one with an affected sibling). Most of the children presented because of poor vision, but one child presented because the parents had noted iridodonesis. The average preoperative vision was $0 \cdot 15(6 / 40)(20$ eyes). Two children had unilateral surgery and the rest bilateral. All children had a lensectomy via a corneal incision as the initial procedure. The average age at surgery was 69 months. Three children had second surgical procedures, which were all divisions of vitreous strands and performed within three months of the initial procedure.

The average postoperative vision was 0.68 (6/ $8.8)$ (16 eyes). In an average follow-up period of 22.4 months (range 3-65 months) there have been no operative or postoperative complications in these children.

CATARACT AND CLEAR LENS SURGERY

Follow-up. The mean follow-up time for the whole group was 36 months (range 2 weeks to 10.3 years). The mean follow-up times for specific groups are given with the visual acuity results.

Type of surgery. A total of 346 primary lens 
operations were performed. These were either a lensectomy or lens aspiration. Lensectomy involved the removal of the whole lens, complete with anterior and posterior capsules and anterior vitreous with an aspirating/cutting machine via a corneal wound, and a separate infusion port or irrigating cystitome. Lens aspiration comprised the removal of the anterior lens capsule, all lens cortex and nucleus, leaving the posterior capsule intact. Twenty five children with bilateral cataracts had an aspiration of one lens and a lensectomy performed in the other eye (Table I). Children over 18 months usually had lens aspirations; infants with congenital cataracts had either lensectomies or aspirations, and all those with retrolental abnormalities had lensectomies.

Age at presentation. Bilateral congenital cataracts presented earlier than unilateral abnormalities (Table II). The graph (Fig 1) shows that among babies with dense bilateral congenital cataracts presenting from within the UK there has been a slight trend towards earlier presentation. The years with the earliest average age of presentation were 1983 and 1986. The time between when symptoms were first noticed and presentation at hospital was at a minimum in 1983 ( 1.25 months).

The mean age at presentation of babies with a family history of cataracts was $4 \cdot 1$ months (17 those without a family history was 4.6 months (70 children).

Reasons for presentation. The primary symptom noted in each case is given in Table III. Poor vision was the commonest reason for presentation, especially in older children. Babies with congenital cataract tended to present with leucocoria or nystagmus. Thirty eight children's lens abnormalities were detected by a routine test; in some this was because cataracts were suspected (for example, in Down's syndrome or family history of cataracts), but in 26 cases the cataracts were a surprise finding at a neonatal check, school test, or admission to hospital for an unrelated condition.

Presenting signs. Nystagmus or random eye movements were the commonest presenting signs (Table IV). Microphthalmos varied in degree from quite severe forms with horizontal corneal diameters often less than $8 \mathrm{~mm}$ associated with PHPV to eyes just less than normal size. Twenty seven children had shallow or flat anterior chambers. These are the cases of PHPV, RLF, and other retrolental abnormalities. Esotropia was commoner in the congenital cataract group, while exotropia was commoner in the juvenile age group.

Diagnosis. In Table V 'simple' refers to an TABLE III Reason for presentation in 212 children with cataract or retrolental abnormalities

\begin{tabular}{lr}
\hline Poor vision & 45 \\
Routine test & 38 \\
Leucocoria & 31 \\
Squint & 26 \\
Nystagmus & 19 \\
Trauma & 12 \\
Painful glaucoma & 4 \\
Microphthalmos & 4 \\
Photophobia & 3 \\
Combination of above & 40
\end{tabular}
TABLE IV Presenting signs in 212 children with cataract and retrolental abnormalities

$$
\begin{aligned}
& \text { Nystagmus/random eye movements } \\
& \text { Esotropia } \\
& \text { Microphthalmos } \\
& \text { Shallow/flat anterior chamber } \\
& \text { Exotropia } \\
& \text { Retrolental mass (9 ROP) } \\
& \text { Retinopathy (6 rubella) } \\
& \text { Band keratopathy } \\
& \text { Posterior lenticonus } \\
& \text { Opaque cornea }
\end{aligned}
$$
children). The average age at presentation of

TABLE V Diagnosis in 212 children with cataracts and retrolental abnormalities

\begin{tabular}{lr}
\hline Simple bilateral congenital cataracts & 59 \\
Simple unilateral congenital cataract & 13 \\
Simple infantile cataracts & 15 \\
Simple juvenile cataracts & 29 \\
Rubella syndrome & 21 \\
Trauma & 12 \\
Retrolental fibroplasia & 12 \\
Severe PHPV & 9 \\
Down's syndrome & 9 \\
Retinal dysplasia & 8 \\
Uveitis & 6 \\
Anterior chamber cleavage syndrome & 4 \\
Norrie's disease & 2 \\
Cerebro-oculo-facial-skeletal syndrome & 2 \\
Lowe's syndrome & 2 \\
Systemic steroid induced & 1 \\
Retinal dystrophy & 1 \\
Diabetes & 1 \\
Radiation & 1 \\
Galactosaemia & 1 \\
Diagnosis uncertain & 4 \\
\hline
\end{tabular}

TABLE VI Positive family history

Autosomal dominant

Probable autosomal recessive

$\mathrm{X}$-linked recessive (Norrie's)

TABLE VII Positive family history

\begin{tabular}{lrr}
\hline & & $\%$ \\
\hline Simple bilateral congenital & $20 / 59$ & 34 \\
Infantile & $2 / 15$ & 13 \\
Juvenile & $9 / 29$ & 31 \\
\hline
\end{tabular}

except perhaps microphthalmic, eye. In 'congenital' cases the cataracts were severe enough to cause visual deprivation from birth. In 'infantile' cases the visual deprivation occurred later than this but before the second birthday. These children had apparently normal fixation and no nystagmus in early infancy. In 'juvenile' cases the onset of the apparent visual deprivation occurred after the second birthday.

Four of the cases of uveitis and the systemic steroid induced case had juvenile rheumatoid arthritis. The child with the radiation induced cataract had been treated for leukaemia. Overall, only $56 \%$ of the cases were simple cataracts.

Family history. Table VI shows the inheritance pattern in 42 cases (of 212) in which there was a positive family history. There was a negative family history in 162 cases and in eight the family history was not recorded. In the probable autosomal recessive group the parents were first cousins in seven cases, in another three cases there was no consanguinity but at least two siblings of different sexes had cataracts, and two occurred in known autosomal recessive syndromes. All the cases with a positive family history were bilateral. The percentage of simple cases with a positive family history is shown in Table VII.

Indications for surgery. $86 \%$ of the children were operated on to improve vision (Table VIII), though some had a poor visual prognosis from the outset - for example, because of retinal dystrophy, Peter's anomaly. Twenty cases underwent clear lens surgery to treat or prevent angle closure glaucoma. In 10 cases the leucocoria was a cosmetic problem.

Operative complications. The operative complications are set out in Table IX. Vitreous 
TABLE VIII Indications for surgery

\begin{tabular}{lr}
\hline & Patients \\
\hline To improve vision & 182 \\
To avoid glaucoma & 14 \\
To treat glaucoma & 6 \\
Cosmetic & 10 \\
Total & 212 \\
\hline
\end{tabular}

haemorrhage was the commonest but all of these cleared spontaneously. There was one case in which $30 \%$ saline was used as the intraocular irrigating fluid, causing retinal necrosis.

Postoperative complications. Seventeen postoperative complications occurred in 16 patients (Table X), giving a complication rate of $4 \cdot 6 \%$. Of the five children who developed a retinal detachment all had particularly unusual features. In one there had been a previous retinal detachment in the other (phakic) eye. Another child had ichthyosis and had had postoperative endophthalmitis in the other eye. A third child had chronic uveitis, a fourth had Lowe's syndrome, and a fifth was severely mentally retarded and institutionalised. In the last case the detachment was detected three years after surgery, and trauma from head banging was suspected.

Both cases of endophthalmitis were apparent on the first postoperative day. The infecting organisms were Staphylococcus aureus and Staph. epidermidis. Both the corneal complications occurred in contact lens wearers. The case of persistent inflammation was in a child with rubella cataract.

Strabismus is not mentioned in Table $\mathrm{X}$. While it was universal in the congenital cases and a little less frequent in later onset cases, the squint was often present preoperatively and therefore could not be considered a postoperative complication. Some degree of posterior capsular opacification was present postoperatively in almost all the cases which had lens aspirations, but not all were of a degree to require capsulotomy.

TABLE X Postoperative complications

\begin{tabular}{ll}
\hline & Eyes \\
\hline Retinal detachment & 5 \\
Chronic aphakic glaucoma & 4 \\
Acute glaucoma & 2 \\
Endophthalmitis & 2 \\
Cystoid macula oedema & 1 \\
Corneal abscess & 1 \\
Corneal ulcer & 1 \\
Persistent inflammation & 1 \\
\hline
\end{tabular}

TABLE XI Secondary procedures - aspiration group

\begin{tabular}{lc}
\hline & Eyes \\
\hline Posterior capsulotomy & 37 \\
Ant. seg. revision & 10 \\
Examination under anaesthetic & 7 \\
Squint surgery & 5 \\
Removal of sutures & 4 \\
Resuture of wound & 2 \\
Retinal detachment & 1 \\
Peripheral iridectomy & 1 \\
Cyclocryotherapy & 1 \\
\hline
\end{tabular}

TABLE XIII Visual results versus age at surgery in bilateral congenital cataracts

\begin{tabular}{lllll}
\hline Age at surgery (months) & $1-2$ & $3-4$ & $5-6$ & $7-12$ \\
\hline Mean vision & $0 \cdot 32(6 / 18)$ & $0 \cdot 18(6 / 33)$ & $0 \cdot 23(6 / 26)$ & $0 \cdot 25(6 / 24)$ \\
Vision $>6 / 24$ & 5 & 2 & 4 & 3 \\
Number & 6 & 7 & 63 & 6 \\
Mean follow-up (months) & 48 & 67 & 63 & 57 \\
\hline
\end{tabular}

TABLE XII Secondary procedures - lensectomy group

\begin{tabular}{lc}
\hline & Eyes \\
\hline Examination under anaesthetic & 30 \\
Ant. seg. revision & 14 \\
Squint surgery & 8 \\
Capsulotomy & 5 \\
Removal of sutures & 5 \\
Division of synechiae & 2 \\
Release of incarcerated iris & 1 \\
Cyclocryotherapy & 1 \\
\hline
\end{tabular}

TABLE XIV Visual results - infantile cataracts

\begin{tabular}{ll}
\hline Mean visual acuity & $0 \cdot 45(6 / 13)$ \\
Mean age at surgery & $22 \cdot 6$ months \\
Mean follow-up & $56 \cdot 9$ months \\
Number & 10 children \\
\hline
\end{tabular}

Secondary procedures. Overall, 128 subsequent procedures were performed on 82 eyes of 72 patients. Forty one of $109(38 \%)$ eyes which had an aspiration had secondary procedures, the most common being a posterior capsulotomy (Table XI). In the lensectomy group 37 of 237 eyes $(16 \%)$ had secondary procedures, with examination under anaesthetic being the commonest type (Table XII). This was usually performed to exclude glaucoma or infection.

The cases requiring anterior segment revision include those in which a vitreous strand was identified in the wound, posterior synechiae had formed, lens remnants were left, or the anterior chamber became shallow.

\section{Visual results}

Bilateral congenital cataract. Table XIII shows the visual results in the better eye in cases of simple bilateral congenital cataract according to the age at surgery. Aphakia was corrected with contact lenses within one or two weeks of surgery, and only a minority (15\%) discontinued lens wear. The average visual acuity was better when surgery was performed within the first two months of life, though the difference was not statistically significant $\left(\chi^{2}\right.$ test, $\left.p=0 \cdot 23\right)$. In most cases strabismus was present, and the strabismic eye had a more marked degree of amblyopia.

Unilateral congenital cataract. These infants were optically corrected with a contact lens, and sound eye occlusion was begun immediately after surgery. The occlusion was of the order of $90 \%$ of the waking hours for the first few weeks and then gradually reduced, but in most cases the occlusion later become unreliable and rather haphazard.

Only six children were old enough to read the Snellen chart. Of these one child achieved 6/24 vision. This child was remarkable in that it was operated on at 7 weeks and the parents were compliant with the occlusion therapy. The other five were all poorly compliant of occlusion despite encouragement and explanation from medical, optometrical, and orthoptic staff, and all had corrected vision of 2/60 or worse. We consider that in the discussion with the parents of prospective surgery for unilateral congenital cataract mention should be made of the high non-compliance rate with the occlusion regimen.

Infantile cataract. Table XIV shows that these children achieved good visual results in the better eye. All these infants had apparently good 
TABLE XV Visual results-juvenile cataracts

\begin{tabular}{lr}
\hline Mean visual acuity & $0 \cdot 68(6 / 9)$ \\
Mean age at surgery & 82 months \\
Mean follow-up & 32 months \\
Number & 21 children \\
\hline
\end{tabular}

TABLE XVI Visual results - trauma

\begin{tabular}{lc}
\hline Mean visual acuity & $0 \cdot 37(6 / 16)$ \\
Mean age at surgery & 82 months \\
Mean follow-up & 21.5 months \\
Number & 9 children \\
\hline
\end{tabular}

fixation prior to the development or progression of the cataracts. They were all bilateral.

Fuvenile cataract. The children whose cataracts developed after the age of 2 years achieved good visual results. All but three of these cases were bilateral, and the acuities shown are in the better eye (Table XV).

Traumatic cataract. All the trauma cases were unilateral, so that in some amblyopia was a problem. The visual results are given in Table XVI.

\section{Discussion}

Just over half the children in this study had simple cataracts. In $44 \%$ the cataract was associated with a congenital syndrome or ocular anomaly or was a secondary cataract. The heterogeneity of the diagnoses reflects the referral pattern of the Hospital for Sick Children, which functions as a tertiary referral centre. About a third of simple cases in our study were familial. This compares with more than $55 \%$ in another London study ${ }^{1}$ and underlines the point that most inherited cases are simple; our figures are biased by the large proportion of complicated cases. The high incidence of retrolental abnormalities is also reflected in vitreous haemorrhage being the commonest operative complication.

It is regrettable that the mean ages of presentation in bilateral congenital cataracts with and without a family history are similar $(4 \cdot 1$ and $4 \cdot 6$ months). Infants born into families with a history of cataracts should be prime candidates for early detection and surgery if necessary. Reminders and encouragement for an early check should be given by the ophthalmologist and paediatrician at every opportunity.

While congenital rubella was not uncommon in the early years of the study, in recent years most cases with this syndrome have been referred from abroad. With the availability of the measles-mumps-rubella vaccine in the UK cataracts due to rubella embryopathy are now entirely preventable. If rubella is considered as a possible aetiology, rubella antibodies should be measured on maternal and infant serum. The lens material removed at surgery should be cultured to grow the virus. The virus may be grown from children up to 4 years of age. In cases of congenital rubella we use steroid drops prior to surgery, subconjunctival steroids at operation, and frequent steroid drops after surgery.

Only one case of cataract due to galactosaemia was operated on. This is because, even though galactosaemia is high on every list of causes of congenital cataracts, the cataracts usually dis- appear within days of beginning a lactose-free diet.

No cases of corneal endothelial decompensation were noted. At present all children undergoing lens surgery at The Hospital for Sick Children have photographs taken of the corneal endothelium at each subsequent procedure. The results of this study will be published in due course.

For the children in this study those with dislocated lenses and infantile and juvenile cataracts had very good visual outcomes. In the past, surgery for dislocated lenses had a high risk of complication and was not to be undertaken lightly. ${ }^{23}$ Modern surgical techniques have changed the indications for operating on a child with a dislocated lens. ${ }^{4}$ We believe that a child should have the lens removed and wear aphakic correction as soon as the level of vision is inadequate for normal life or the situation of the lens is such as to make amblyopia likely.

Children with simple bilateral congenital cataracts removed and optically corrected at an early age can expect reasonable vision. If $6 / 24$ vision is taken as a level above which a child should be able to be educated at a normal school with minimal extra help, five of six children in this early surgery group achieved better than this level of vision. Earlier surgery and correction are probably associated with better visual results, though our numbers were small and the results were not statistically significant. By the time the visual defect is obvious to the parents, the optimum time for surgery is likely to have passed. Consequently, to detect the cases early enough a test for obstruction of the red reflex on ophthalmoscopy must be part of the checking of every newborn and 6-week baby. ${ }^{5-7}$ Ophthalmologists should encourage and instruct their paediatric and general practice colleagues to include this.

In children with unilateral congenital cataracts the visual results are disappointing and are not comparable with those reported by some other authors. ${ }^{89}$ Opaque contact lenses ${ }^{10}$ have been tried without success in our department, and we rely on a rigorous occlusion programme. Nevertheless dense amblyopia remained the norm. The cost effectiveness of surgery in these unilateral cases remains controversial, especially since a good visual result may lead to diplopia. ${ }^{11}$

Because of the advent of the horizontally mounted, vertically aimed YAG laser, we intend in future to perform a lens aspiration followed by laser capsulotomy, in all cases with the exceptions of ectopia lentis, cases in which an aspiration on the other eye was difficult or complicated, posterior lenticonus, and PHPV and other retrolental abnormalities. In these cases lensectomy will continue to be the preferred procedure. The main advantage of an aspiration is that implantation of a posterior chamber intraocular lens remains an option which can be considered in the future. There are also the theoretical advantages of a lesser risk of retinal detachment and cystoid macular oedema. One infant in the study with a limited life expectancy, and considerable systemic problems related to the cerebro-oculo-facial-skeletal syndrome, had an intraocular lens implanted at the primary 
operation. However, because of the rapidly changing refraction in infancy, the unknown long term effects of intraocular lens implantation, and the high acceptance rate of contact lens use, we do not recommend intraocular lens implantation at present.

We thank Mr Simon Brown, of the Medical Illustration Department, and Mrs Ailsa Abel, of the Medical Records Department, for their assistance. This study was partly funded by the Iris Fund and the Help a Child to See charity.

1 Burn EC, Jones RB. Long term management of congenital cataracts. Arch Dis Child 1985; 60: 322-5.

Chandler PA. Choice of treatment in dislocation of the lens Arch Ophthalmol 1964; 71: 765-86.

3 Maumenee IH. The eye in Marfan syndrome. Trans Am Ophthalmol Soc 1981; 79: 684-733.
4 Reese PD, Weingeist TA. Pars plana management of ectopia lentis in children. Arch Ophthalmol 1987; 105: 1202-4.

5 Taylor D, Rice N. Annotation. Congenital cataract, a cause of preventable child blindness. Arch Dis Child 1982; 57: 165-7.

6 Rice NSC, Taylor D. Congenital cataract: a cause of preventable blindness in children. BrMed f 1982; 285: 581-2.

7 Taylor D. Cataracts in children (Editorial). $\mathcal{f} R$ Soc Med 1985; 78: $1-3$.

8 Robb RM, Mayer DL, Moore BD. Results of early treatment of unilateral congenital cataracts. $\mathcal{F}$ Pediatr Ophthalmol Strabismus $1987,24: 178-81$.

9 Drummond GT, Scott WE, Keech RV. Management of monocular congenital cataracts. Arch Ophthalmol 1989; 107: 45-51.

10 Mayer DL, Moore B, Robb RM. Assessment of vision and amblyopia by preferential looking tests after early surgery for unilateral congenital cataracts. $\mathcal{F}$ Pediatr Ophthalmol Strabismus 1989; 26: 61-8.

11 Pratt-Johnson JA, Tillson G. Unilateral congenital cataract: Binocular status after treatment. 7 Pediatr Ophthalmol Strabismus 1989; 26: 72-5. 